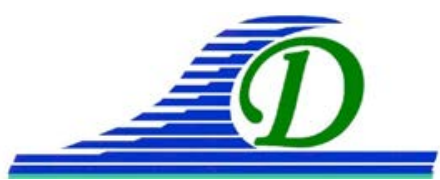

XIII ${ }^{\text {èmes }}$ Journées Nationales Génie Côtier - Génie Civil Dunkerque, 2-4 juillet 2014

DOI:10.5150/jngcgc.2014.107 @ Editions Paralia CFL

disponible en ligne - http://www.paralia.fr - available online

\title{
Valorisation des fines de dragage en Manche
}

\section{Mickaël BERTRAND ${ }^{1}$, Philippe DHERVILLY ${ }^{1}$, Daniel LEVACHER ${ }^{2}$}

\section{SEDIGATE S.A.S., 17 rue Claude Bloch, BP 25 102, 14000 Caen Cedex, France.}

m.bertrand@sedigate.com; contact@sedigate.com

2. Université de Normandie, Unicaen, Laboratoire Morphodynamique Continentale et Côtière, UMR 6143 M2C, 24 rue des Tilleuls, F-14000 Caen, France.

daniel.levacher@unicaen.fr

\section{Résumé :}

L’étude porte sur des sédiments provenant de la mer Manche et plus précisément des ports de Dieppe, de Fécamp et du Havre. Les trois sédiments ont été mélangés pour ne faire qu'un seul matériau à valoriser.

Plusieurs formulations à base de liants pouzzolaniques ont été testées. Des essais mécaniques de résistance à la compression simple ont été effectués pour en situer les performances. Par ailleurs, chacune des formulations doit aussi respecter à la fois des critères économiques et écologiques. Une fois validées, ces formulations permettent d'aboutir à la stabilisation renforcée des sols de surface pour la réalisation de voies vertes, de vélo-routes ou d'allées naturelles.

Ces liants, développés à partir de la combustion de sous-produits industriels, sont à la base de plusieurs procédés, comme les procédés ECOVOIE ${ }^{\circledR}$ et ACTiV.SOL ${ }^{\circledR}$, permettant l'utilisation des sédiments comme matériaux nobles.

Mots clés : Valorisation, Stabilisation, Solidification, Sédiment, Boue industrielle, Liant.

\section{Introduction}

La société SEDIGATE explore depuis sa création les voies de co-valorisations possibles des matériaux fins en technique routière. Les matériaux en question sont des sédiments issus de trois ports différents, situés sur le littoral de la mer Manche. Ils ont fait l'objet d'échantillonnages aux ports de Dieppe, de Fécamp et du Havre. Les trois sédiments sont homogénéisés pour ne faire plus qu’un seul et même matériau de base à valoriser. La valorisation de ce type de matériau passe par la stabilisation et la solidification de ce dernier. Plusieurs formulations robustes à base de sous-produits industriels, i.e. à base de cendres volantes, composant un liant à prise pouzzolanique, sont expérimentées. Pour en situer les performances mécaniques, des essais de résistance à la compression simple ont été menés sur différents mélanges.

L'objectif est de mettre en place par la suite un traitement en masse des matériaux fins étudiés. 


\section{Méthodologie}

\subsection{Caractéristiques physico-chimiques}

Toute valorisation nécessite la détermination des caractéristiques physico-chimiques de ces matériaux. Elles sont reportées dans le tableau 1. Les trois sédiments sont ensuite homogénéisés pour ne faire qu'un seul matériau à traiter. Cette étape permet de simuler un traitement de masse de plusieurs sédiments d'origine diverse. L'homogénéisation dans notre cas de figure est effectuée, avec un malaxeur normalisé de type mortier, dans des proportions égales pour chaque sédiment, à savoir un tiers des sédiments de Dieppe, un tiers de ceux de Fécamp et un tiers en provenance du Havre.

Tableau 1. Propriétés physico-chimiques des sédiments de Dieppe, de Fécamp et du Havre.

\begin{tabular}{|c|c|c|c|c|c|}
\hline Paramètre & Méthodes & $\begin{array}{l}\text { Sédiment } \\
\text { Dieppe }\end{array}$ & $\begin{array}{l}\text { Sédiment } \\
\text { Fécamp }\end{array}$ & $\begin{array}{l}\text { Sédiment } \\
\text { Havre }\end{array}$ & $\begin{array}{l}\text { Sédiment } \\
\text { moyen }\end{array}$ \\
\hline Matière organique (\%) & Perte au feu à $550^{\circ} \mathrm{C}$ & 7.0 & 5.0 & 2.5 & 4.8 \\
\hline \multirow{7}{*}{$\begin{array}{l}\text { Nature } \\
\text { Granulométrique } \\
(\%)\end{array}$} & Tamisage et laser & & & & \\
\hline & Argiles & & & & \\
\hline & $0<d<2 \mu m$ & 3 & 8 & 5 & 5 \\
\hline & Limons : & & & & \\
\hline & $2<d<63 \mu m$ & 88 & 48 & 80 & 72 \\
\hline & Sables : & & & & \\
\hline & $63 \mu \mathrm{m}<d<2 \mathrm{~mm}$ & 9 & 44 & 15 & 23 \\
\hline
\end{tabular}

Les caractéristiques du matériau final correspondent aux moyennes des valeurs de chaque paramètre obtenues pour chaque sédiment. La teneur en matière organique du matériau moyen est considérée comme faible, elle est égale à 4.8\%. Généralement, nous pouvons considérer qu'un matériau a une teneur en matière organique élevée à partir du moment où celle-ci est supérieure à 5\% (TROALEN et al., 1996). L'ajout d'un additif sera nécessaire pour neutraliser la matière organique présente ici en faible quantité.

L'analyse granulométrique révèle un matériau moyen majoritairement limoneux, les particules de dimensions inférieures à $63 \mu \mathrm{m}$ représentent $72 \%$ des constituants.

L’étude menée sur ces sédiments propose l'utilisation de liants pouzzolaniques pour un dosage inférieur à $10 \%$ pour la stabilisation et solidification.

\subsection{Mise en œuvre des formulations et évaluation des performances mécaniques}

De récentes études menées en laboratoire et sur sites ont déjà abouti à de bonnes performances quant à l'utilisation de liants pouzzolaniques pour une valorisation de sédiments marins en technique routière (BEHMANESH, 2008 ; SILITONGA et al., 2010 ; MIRAOUI, 2010 ; OBANA et al., 2010). La stabilisation/solidification du 


\section{XIII ${ }^{\text {èmes }}$ Journées Nationales Génie Côtier - Génie Civil \\ Dunkerque, 2-4 juillet 2014}

matériau moyen composé des sédiments de Dieppe, de Fécamp et du Havre a porté sur l'étude de mélanges sédiment-liant-additif dont les formulations sont données dans le tableau 2. Ces mélanges ont donné lieu à la confection d'éprouvettes cylindriques, de 34 $\mathrm{mm}$ de diamètre et d'un élancement de 2 . La teneur en eau $\mathrm{W}$ des sédiments incorporés dans les mélanges était de $25 \%$, soit une siccité de $80 \%$. Nous rappelons que la teneur en eau W (\%) et la siccité S (\%) s'expriment comme suit :

$W=\frac{W_{e}}{W_{s}}$ et $S=\frac{W_{e}}{W_{t}}$

avec $W e, W s, W t$ comme étant les masses respectives d'eau, de matière sèche et totale.

L'ensemble des échantillons a été conservé sur paillasse à l'air libre à une température voisine de $20^{\circ} \mathrm{C}$. Des essais de résistance en compression simple ont été réalisés pour l'ensemble des mélanges étudiés aux durées conventionnelles de conservation de 7, 14 et 28 jours. Les teneurs en liants et additif données dans le tableau 2 sont exprimées en pourcentage de la masse du sédiment sec. Le liant pouzzolanique est une cendre volante silico-alumineuse capable de stabiliser des sables et des sédiments fins de manière durable. L'additif utilisé est un hydroxyde de calcium, plus connu sous le nom de chaux. La chaux est utilisée pour réduire l'interférence de la matière organique sur les réactions pouzzolaniques des cendres volantes, et permettre aussi d'activer ces réactions. Des formulations de mélange ont été testées et comparées entre elles (tableau 2). L'indicateur mécanique déterminant évalué est la résistance mécanique à la compression simple $R c$ dont le seuil retenu est de $1 \mathrm{MPa}$ à 28 jours de séchage (GTR SETRA-LCPC, 2000). Ce seuil permettra de valider les formulations utilisées.

Tableau 2. Formulations proposées.

\begin{tabular}{lll}
\hline Références & Liant pouzzolanique (\%) - LP & Additif (\%) - A \\
\hline Témoin T & 0 & 0 \\
Formulation F1 & 5 & 1 \\
Formulation F2 & 6 & 1 \\
Formulation F3 & 7 & 1 \\
Formulation F4 & 8 & 1 \\
\hline
\end{tabular}

\section{Résultats}

Les résultats des essais de compression simple sur les mélanges ont permis d'établir les constats suivants (figure 1). L'évolution dans le temps des résistances $R c$ des formulations F1, F2, F3 et F4 présente une tendance classique avec des valeurs croissantes durant 28 jours de séchage. Le mélange Témoin qui est sans liant et additif, montre l'efficacité des différentes formulations étudiées. Elles atteignent respectivement 2.0, 2.6, 2.9 et 3.0 MPa à 28 jours. Les formulations ne présentent pas de palier de résistance à 28 jours. Les formulations $\mathrm{F} 2$, F3 et $\mathrm{F} 4$ présentent des valeurs de $R c$ 
nettement supérieures à 1 MPa dès 7 jours. La formulation F1 possède quant à elle une résistance voisine de $1 \mathrm{MPa}$ à 7 jours.

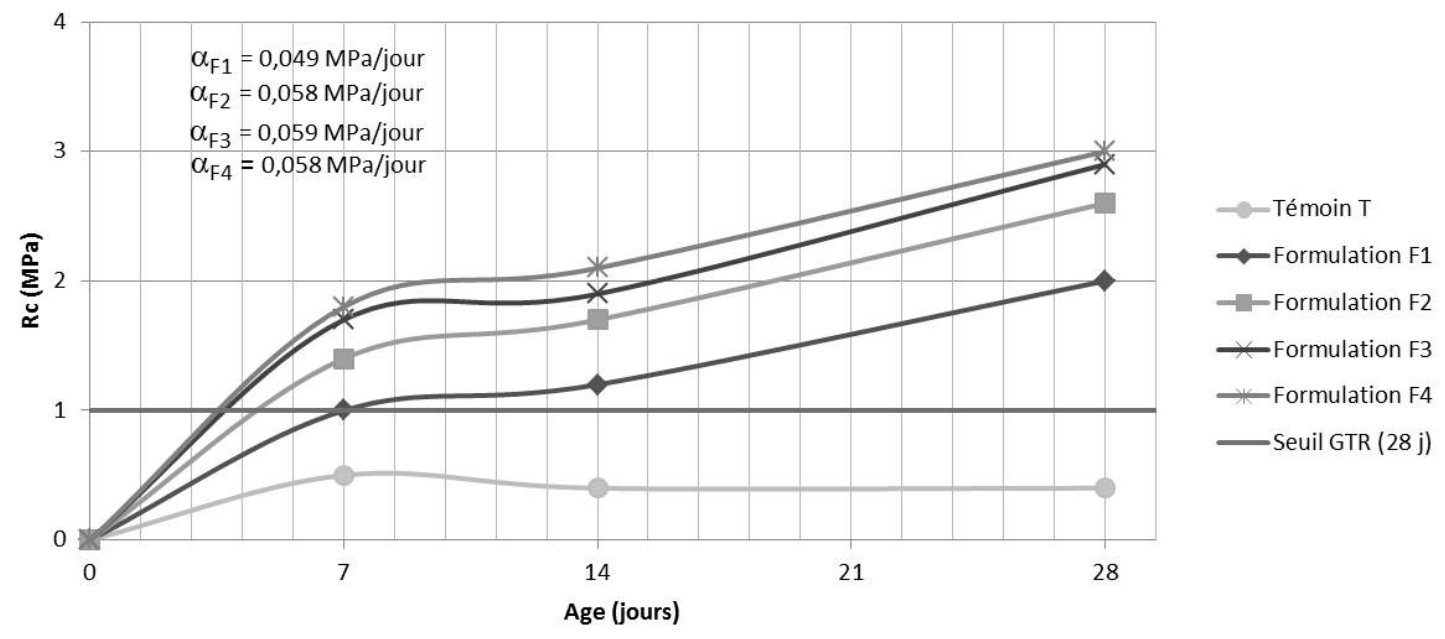

Nota : $\alpha_{F i}$ sont les pentes des droites ajustées pour chaque formulation

Figure 1. Evolution des résistances à la compression simple

L'ensemble des formulations fournissent des résistances à la compression simple suffisantes dès 14 jours de séchage. En effet, elles sont supérieures au seuil de résistance fixé à $1 \mathrm{MPa}$ à 28 jours de séchage pour d'éventuelles circulations d'engins de chantier. Finalement, la formulation retenue pour une application en technique routière est la formulation F1 qui respecte les caractéristiques souhaitées, avec une incorporation de liant au plus faible dosage dans le matériau sans additif.

La figure 2 montre l'évolution des rapports des différentes valeurs de Rc des formulations étudiées par rapport à la valeur de $R c$ obtenue avec l'échantillon Témoin (a) et la formulation F1 (b). On remarque d'une part l'effet du liant LP et de l'additif A dans les mélanges : plus le pourcentage de liant est élevé et plus le rapport croit. D’autre part, ces rapports calculés par rapport à la formulation F1 montrent directement l'effet du liant pouzzolanique.

La figure 3 montre l'évolution des différentes valeurs de $R c$ des formulations F1, F2, F3 et F4 à 28 jours de séchage. Les données confirment la tendance observée précédemment, i.e. une évolution croissante de $R c$ avec la quantité de liants pouzzolaniques ajoutée dans les mélanges. Mais une tendance asymptotique amène à dire qu'un pourcentage maximal de liants serait atteint vers $10 \%$. On peut aussi à partir d'un ajustement polynomial, en considérant la valeur de l'échantillon Témoin (l'effet de l'additif négligé), déterminer le taux minimal de liant pouzzolanique à ajuster pour obtenir au moins $1 \mathrm{MPa}$ de résistance. 


\section{XIII ${ }^{\text {èmes }}$ Journées Nationales Génie Côtier - Génie Civil \\ Dunkerque, 2-4 juillet 2014}

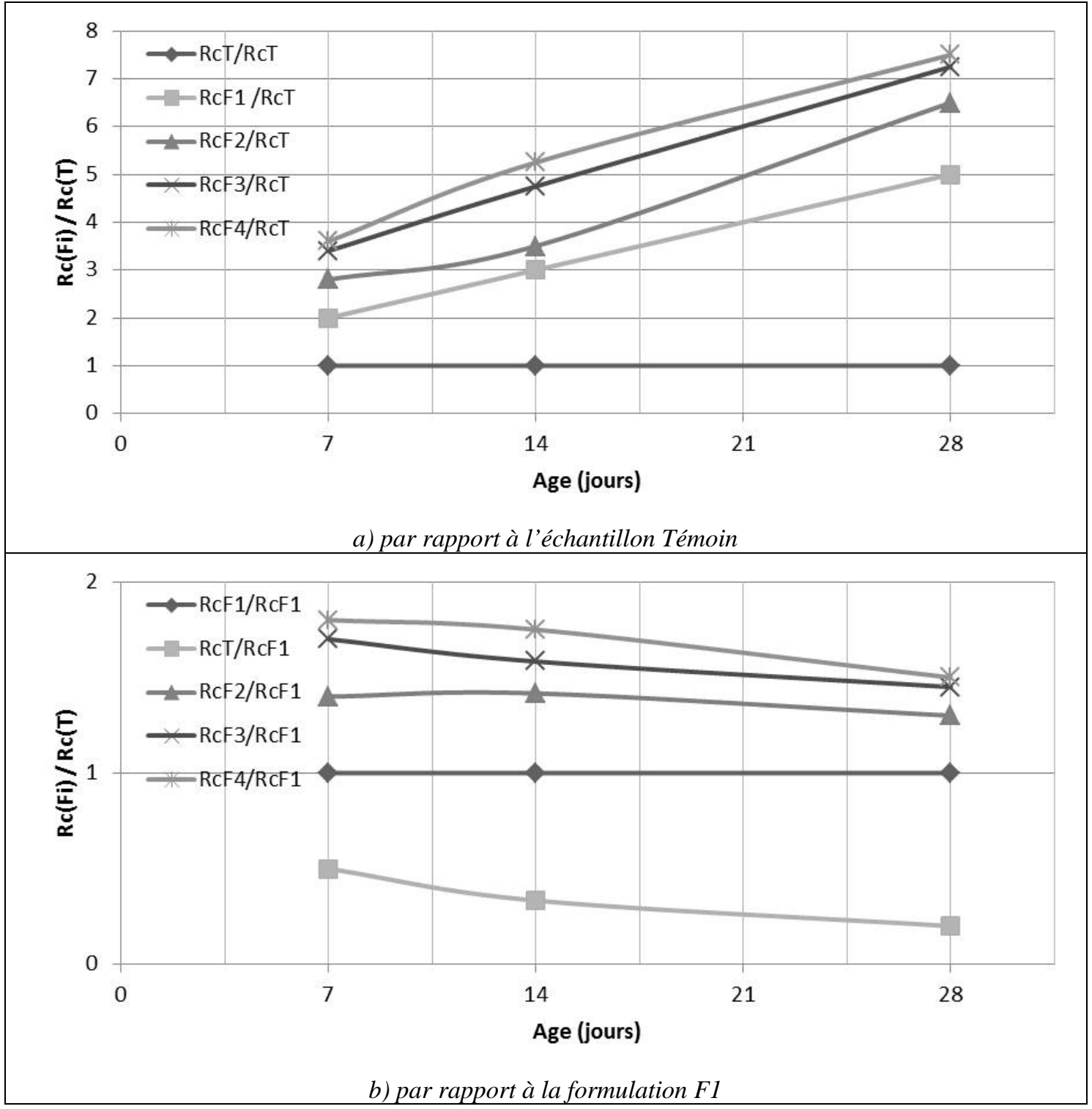

Figure 2. Valeurs comparées de Rc des différentes formulations en fonction temps.

Si l'on mène des ajustements linéaires $R c$ en fonction du temps (figure 1), on peut observer l'évolution des pentes i.e. le temps d'accroissement de $R c$ en 7 et 28 jours. L'évolution des pentes pour les formulations F1, F2, F3 et F4 en fonction des pourcentages du liant pouzzolanique ajouté est illustrée à la figure 4.

Même si l'on observe une augmentation de la pente $\alpha$, les valeurs restent très proches et la valeur moyenne est de $0.056 \mathrm{MPa}$ /jour sur une période de cure de 7 à 28 jours. 


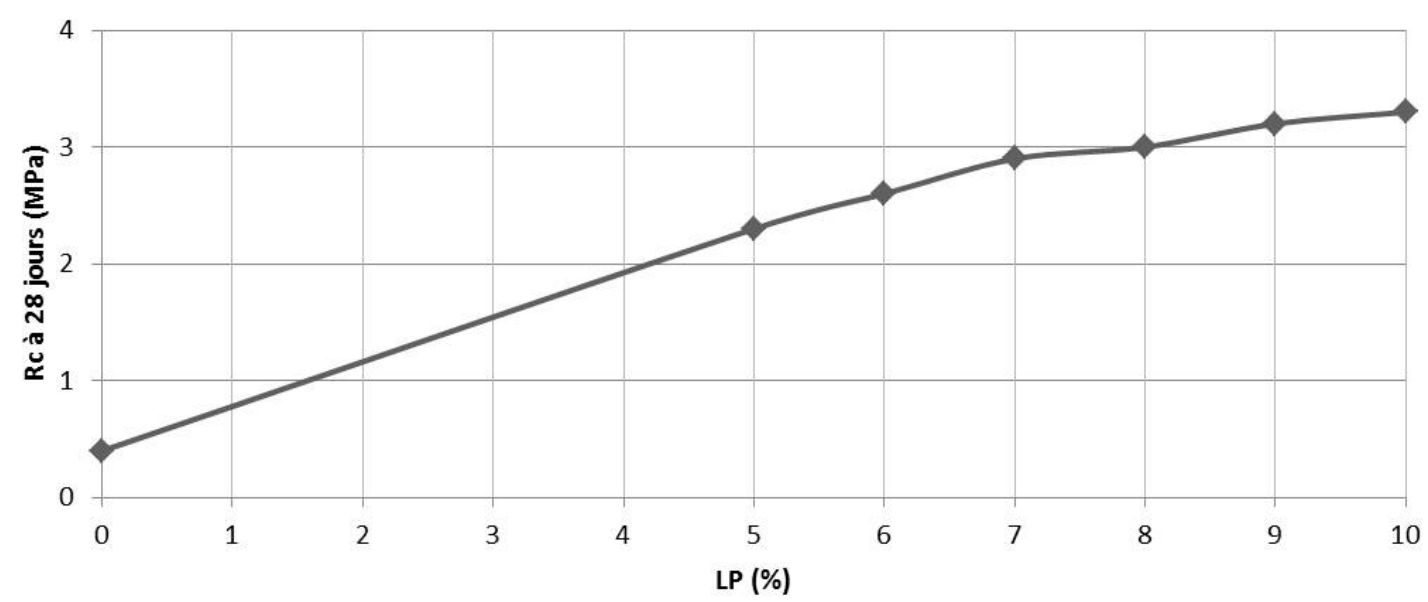

Figure 3. Evolution des valeurs de Rc à 28 jours en fonction du dosage en liants pouzzolaniques (LP).

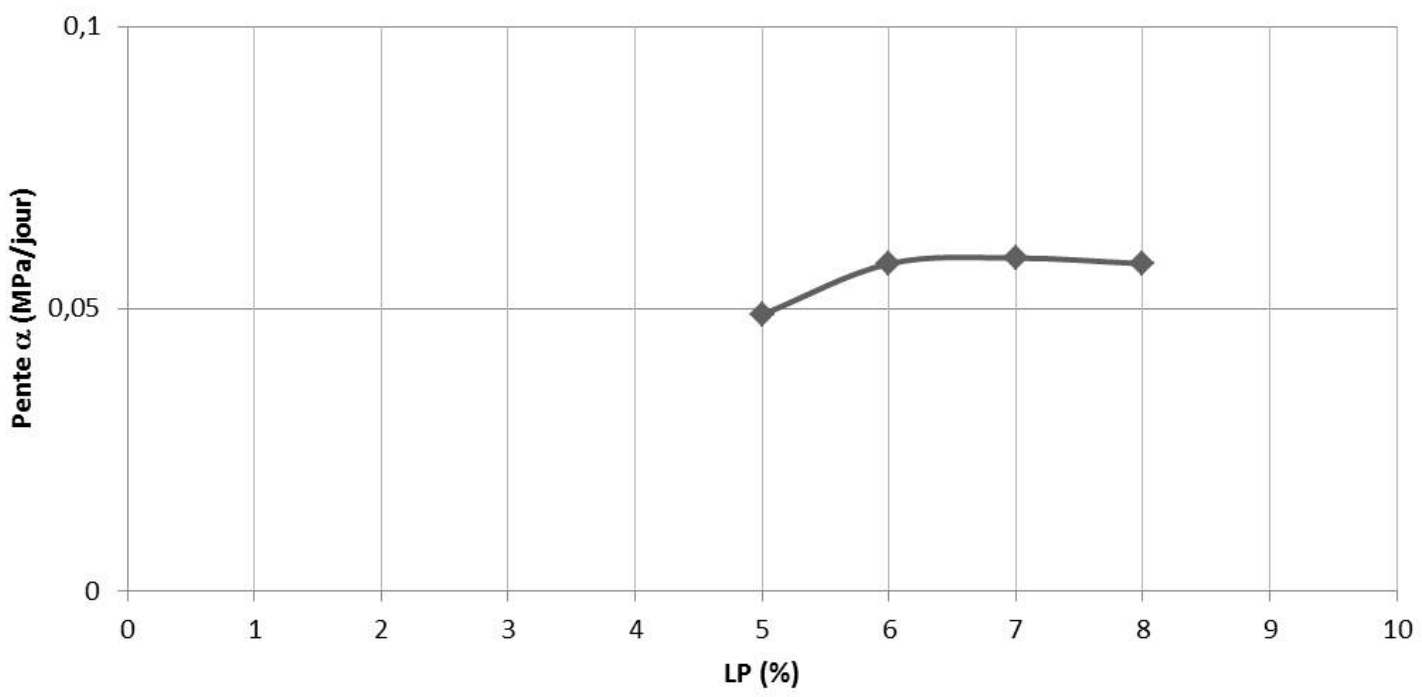

Figure 4. Pentes des différentes formulations en fonction du dosage en liants pouzzolaniques (LP).

\section{Conclusion et perspectives}

Les trois sédiments du littoral de la mer Manche ont servi à établir un seul et même matériau pour l'étude de stabilisation et solidification dans l'optique d'un regroupement de sédiments sur un site de traitement.

La formulation F1 qui incorpore le moins de liant dans le matériau, possède à la fois les critères techniques, économiques et écologiques pour une utilisation des sédiments en technique routière. Elle fournit une résistance à la compression simple suffisante dès 14 


\section{XIII ${ }^{\text {èmes }}$ Journées Nationales Génie Côtier - Génie Civil \\ Dunkerque, 2-4 juillet 2014}

jours de séchage. Cette formulation est à base de cendres volantes qui dégage moins de $\mathrm{CO}_{2}$ qu'une formulation classique à base de ciment. Ce constat est fait par l'observation du bilan carbone réalisé pour la fabrication des deux liants comparés. En effet, un ciment (type CEM II) est fabriqué à base de clinker (65 à 94\%), de cendres volantes (6 à $35 \%$ ) et d'autre constituants secondaires (jusqu'à $5 \%$ ). Le bilan carbone pour la fabrication du ciment est plus élevé que les cendres volantes, puisqu'elles font partie de la composition du ciment (CEM II). Les cendres volantes ont un bilan carbone 3 à 4 fois moins élevé que le ciment pour des injections dans les mélanges équivalentes. La formulation est donc plus écologique qu'une formulation classique à base de ciment.

Cette formulation qui co-valorise un co-produit industriel (liant pouzzolanique) correspond bien à la notion de gestion durable.

Un traitement de masse peut être envisagé pour valoriser en technique routière les différents sédiments fins des ports de Dieppe, de Fécamp et du Havre. Ce traitement de masse est connu par la société SEDIGATE, puisqu'elle réalise des chantiers dont les matériaux de base sont des sédiments mélangé avec du liant. Il existe deux types de procédés : ACTiV.SOL ${ }^{\circledR}$ (en centrale) ou ECOVOIE ${ }^{\circledR}$ (in situ).

Cette étude correspond aux objectifs du programme COVASED (DHERVILLY et al., 2014) destiné à la CO-VAlorisation des SEDiments à l'échelle industrielle par apport de liants et additifs éco-compatibles sur une plateforme de traitement sécurisé. A ce titre, les matériaux entrant sur cette plateforme subissent des prétraitements au préalable (déshydratation, dépollution, ...) et sont par la suite mélangés avant le traitement final pour en faire un matériau de réemploi en routes et remblais.

\section{Références bibliographiques}

BEHMANESH J. (2008). Etude de la durabilité d'un sédiment, traité au ciment et additifs. Thèse de doctorat, Université de Caen, 215 p.

DHERVILLY P., BERTRAND M., THANNEBERGER L., LEVACHER D., HOUISE C., LAFHAJ Z. (2014). Gestion durable des sédiments : le projet COVASED. XIII ${ }^{\text {èmes }}$ Journées Nationales de Génie Côtier - Génie Civil, Dunkerque, 2-4 Juillet 2014. http://dx.doi.org/10.5150/jngcgc.2014.108

GTR SETRA-LCPC. (2000). Traitement des sols à la chaux et/ou aux liants hydrauliques, Application à la réalisation des remblais et des couches de forme, $108 \mathrm{p}$. MIRAOUI M. (2010). Mise en œuvre d'une démarche de prétraitement et de traitement des sédiments de dragage en vue d'une valorisation dans le génie civil. Thèse de doctorat, Université de Lille, $210 \mathrm{p}$.

OBANA M., LEVACHER D., DHERVILLY P. (2010). Durability properties of marine sediments stabilized by pozzolan and alkali activated binders. XI ${ }^{\text {èmes }}$ Journées Nationales de Génie Côtier - Génie Civil, Sables d’Olonne, pp 887-894. http://dx.doi.org/10.5150/jngcgc.2010.096-O 
Thème 8 - Gestion et valorisation des sédiments marins

SILITONGA E., LEVACHER D., MEZAZIGH S. (2010). Utilization of fly ash for stabilization of marine dredged sediments. European Journal of Environmental and Civil Engineering, Vol. 14, n 2, pp 253-265. http://dx.doi.org/10.3166/ejece.14.253-265

TROALEN J.P., DURON C., HAGNERE C. (1996). Traitement de vases de l'estuaire de la Seine, $4^{\text {èmes }}$ Journées Nationales Génie Civil Génie Côtier, Dinard, France, pp 369-376. http://dx.doi.org/10.5150/ingcgc.1996.048-T 